\title{
Detachment mechanisms of turbulent non-premixed jet flames at atmospheric and elevated pressures
}

\author{
T.F. Guiberti ${ }^{\mathrm{a}, *}$, W.R. Boyette ${ }^{\mathrm{a}}$, A.R. Masri ${ }^{\mathrm{b}}$, and W.L. Roberts ${ }^{\mathrm{a}}$ \\ ${ }^{a}$ King Abdullah University of Science and Technology (KAUST), CCRC, Thuwal 23955-6900, Saudi \\ Arabia. \\ ${ }^{b}$ School of Aerospace, Mechanical and Mechatronic Engineering, The University of Sydney, NSW, \\ 2006 Australia.
}

Prepared for submission to:

Combustion and Flame

Color figures for online version only.

*Corresponding Author:

Thibault F. Guiberti

Clean Combustion Research Center

4216-WS10, Al-Kindi West

King Abdullah University of Science and Technology (KAUST)

Thuwal 23955-6900, Saudi Arabia

Tel: + 966542492338

E-mail: thibault.guiberti@kaust.edu.sa

\begin{abstract}
The stability limits of a turbulent flame in a practical combustor are important characteristics that influence its performance. The mechanisms controlling the stability limits of turbulent non-premixed flames are examined here in the canonical configuration of a fuel jet in co-flow air. This study focuses on the conditions leading to the detachment of flames from the injector nozzle by means of an experimental parametric study in which pressure ( $1 \leq P \leq 10$ bar), fuel (methane and ethane), nozzle wall thickness $(t=0.20 \mathrm{~mm}, 0.58 \mathrm{~mm}$, and $0.89 \mathrm{~mm})$, jet velocity $\left(0.5 \leq U_{j} \leq 16.5 \mathrm{~m} . \mathrm{s}^{-1}\right)$, and co-flow velocity $\left(U_{c}=0.3 \mathrm{~m} . \mathrm{s}^{-1}, 0.6 \mathrm{~m} . \mathrm{s}^{-1}\right.$, and $\left.0.9 \mathrm{~m} . \mathrm{s}^{-1}\right)$ are varied. It is shown that the mechanism leading to detachment depends on the ratio of the nozzle wall thickness to the laminar flame thickness. If this ratio is smaller than 3, the nozzle is "thin" and type I detachment occurs (flame base stability lifting). In this case, the detachment velocity decreases with pressure and is proportional to the laminar burning velocity. If the ratio is larger than 3, the nozzle is "thick" and type II detachment occurs (local flame extinction lifting). Then, the detachment velocity is controlled by the extinction strain rate. Experiments also show that the Kolmogorov scale of turbulence regulates local flame extinction and type II detachment and a model is proposed to predict detachment for any fuel, pressure, and nozzle wall thickness using the computed extinction strain rate and the Kolmogorov time scale. Finally, the data show that elevating pressure allows stabilizing attached non-premixed jet flames with high Reynolds numbers without the need for complex stabilization strategies such as pilot flames, swirl, or
\end{abstract}


oxygen/hydrogen enrichment. Pressure allows studying flame/turbulence interactions at Reynolds numbers relevant to practical applications while conserving simple configurations amenable to diagnostics and modeling.

Keywords: Jet flame; Elevated pressure; Detachment; Lift-off; Local extinction; Kolmogorov scale 


\section{Introduction}

Flame stability limits refer to a broad range of conditions that disturb the otherwise steady behavior of a flame and such effects can range from lift-off from the burner lip to intermittent blow-off, to transient fluctuations induced by heat release and unsteady pressure feedback. Stability limits or transfer functions are usually employed to characterize relevant aspects of flame stability and determine operational boundaries. Operating within these boundaries is key to maximizing the flame's performance with respect to efficiency, emissions, and safety. Understanding flame stability remains a challenge and the interested reader is referred to a number of detailed reviews [1-5].This paper is concerned with the specific issue of flame detachment or lift-off with the objective of shedding light on the factors controlling this behavior at atmospheric as well as high pressure environments.

Turbulent non-premixed jet flames are either attached to the injector nozzle, lifted, or blown-out [6, 7] and boundaries between these three states are controlled by a number of parameters, including the velocity $U_{j}$. Starting with an attached flame that is not stabilized by other means such as pilot or recirculation, increasing the jet velocity will lead to detachment resulting in either flame lift-off or blow-out. In the case of a lifted flame, if the jet velocity is further increased, the flame eventually undergoes blow-out. Stability limits of turbulent non-premixed jet flames, mostly at atmospheric pressure, have been studied extensively with various theories proposed about the controlling mechanism of detachment and/or stabilization [6, 8-13].

Depending on the nozzle configuration, two distinct flame detachment mechanisms can occur as the jet velocity is increased. These were extensively described by Takahashi and Schmoll [6] in their investigation of turbulent non-premixed jet flames at atmospheric pressure:

- Type I: Flame base stability lifting: In this case, flame detachment occurs when the velocity at the base of the flame exceeds the local maximum burning velocity, considered to be equal to the laminar burning velocity $S_{L}[6]$. This is an aerodynamic mechanism.

- Type II: Local flame extinction lifting: In this case, detachment is triggered by local extinction of the flame front at a breakpoint generally located downstream of the flame base. Flameturbulence interactions here play an important role [6, 13-16].

Based on experimental and numerical observations at atmospheric pressure $[6,8,9]$, it is possible to discriminate between type I and type II detachment mechanisms depending on whether the injector nozzle is "thin" (type I) or "thick" (type II) compared to a representative flame thickness. This characteristic length may be the laminar flame thickness [9] or the quenching distance [6]. With type I detachment, the limiting jet velocity should increase with increasing laminar burning velocity $S_{L}$ or 
decreasing co-flow velocity $U_{c}$. This is because both $S_{L}$ and $U_{c}$ influence the local balance at the flame base. If the nozzle wall thickness is large in comparison to a representative flame thickness (type II), the flame base is anchored in the low velocity region delineated by the recirculation zone in the wake of the nozzle rim. In this case, the flame base is tucked-away and protected from the incoming flow, and hence expected to be fairly insensitive to both $S_{L}$ and $U_{c}$. It was shown in [6] that the detachment velocity in type II detachment is the result of extinction events due to high strain rate experienced by the flame. The detachment velocity is then a function of the extinction strain rate $\kappa_{\text {ext }}$ and is, therefore, a function of the fuel and pressure.

While detachment of turbulent non-premixed jet flames has been studied extensively at atmospheric pressure [6, 8-12], experimental data remain very scarce for higher pressures [17] relevant to practical combustion devices. Some detachment data obtained at elevated pressure is available in a recent study by Bang et al. [17] but the main focus of that study was laminar, transition, and low-Reynolds-number turbulent regimes (up to $\mathrm{Re}=5,500$ ). The present study focuses on the detachment mechanisms of turbulent non-premixed jet flames at high Reynolds number and over a range of pressures extending up to $\operatorname{Re}=48,300$ and $P=10$ bar. Other relevant parameters such as nozzle wall thickness, fuel, coflow velocity, and jet velocity are varied. Increasing pressure allows studying effects of modifying the laminar burning velocity without changing the fuel [18]. In addition, increasing pressure has a large influence on the representative flame thickness [18-20], whether it is the laminar flame thickness or the quenching distance, and is, therefore, a promising way to switch between "thin" and "thick" nozzle regimes without actually changing the nozzle dimensions.

\section{Experimental setup and methods}

Experiments are conducted at pressures up to $P=10$ bar in the high-pressure combustion duct (HPCD) available at the Clean Combustion Research Center in KAUST. Its main features are shown in Fig. 1 and more details are available elsewhere [20-22]. The burner consists of a 0.57-m long

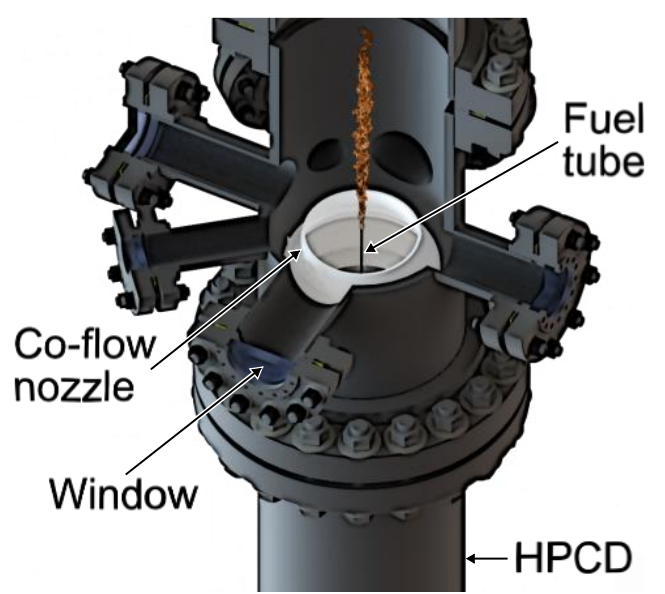

Fig. 1: Schematic of the experimental setup. 
stainless steel tube surrounded by a $0.25-\mathrm{m}$ diameter co-flow nozzle providing an air co-flow of velocities $U_{c}=0.3 \mathrm{~m} . \mathrm{s}^{-1}, 0.6 \mathrm{~m} . \mathrm{s}^{-1}$, or $0.9 \mathrm{~m} . \mathrm{s}^{-1}$. The uniformity of the co-flow velocity at the exit of the co-flow nozzle is within $10 \%$.

Three burner nozzles of different dimensions are examined in this study and these are shown in Fig. 2 . These nozzles feature different inner and/or outer diameters so that three different nozzle wall thicknesses are achieved: $t=0.20 \mathrm{~mm}, 0.58 \mathrm{~mm}$, and $0.89 \mathrm{~mm}$. The two nozzles with $t=0.58 \mathrm{~mm}$ and $0.89 \mathrm{~mm}$ have square exit profiles while the nozzle with $t=0.20 \mathrm{~mm}$ is tapered. The HPCD is aircooled and its pressure is regulated by a dome-loaded back-pressure regulator. Therefore, co-flow air must be supplied continuously to dissipate the flame's thermal power and maintain the desired pressure set-point. Two fuels are examined, namely methane and ethane. For ethane, only the nozzle with the smaller inner diameter (ID $=3.35 \mathrm{~mm}$ corresponding to $t=0.58 \mathrm{~mm}$ ) is used and this allows accommodating for the larger volumetric energy density of ethane and maintain the thermal power within acceptable limits.

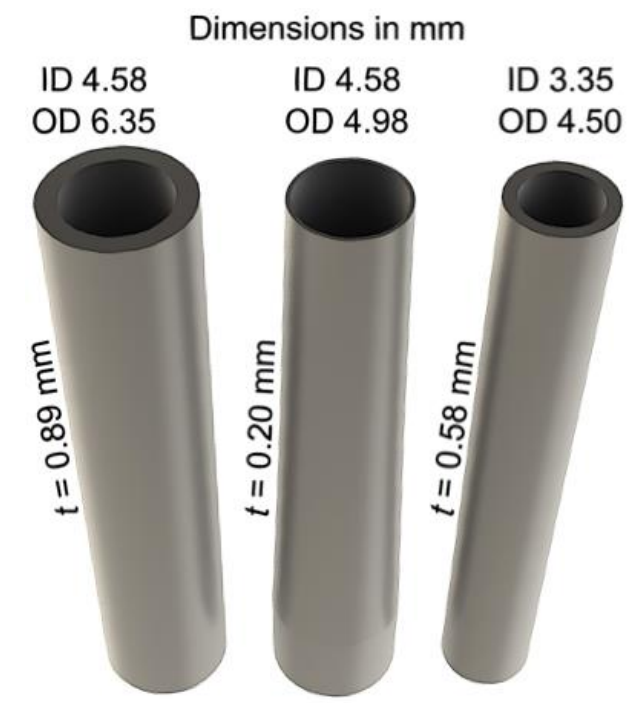

Fig. 2: Schematic of the three nozzles used. 
The fuel mass flow rate is regulated using two thermal mass flow controllers for each fuel (Brooks SLA5851 and SLA5853) featuring two different full scales (49 and 189 slpm for methane and 30 and 114 slpm for ethane) to ensure that metering is realized near the full scale of each device, with optimal accuracy. These mass flow controllers are calibrated with air using a gas flow calibrator (MesaLabs ML-1020) prior to experiments to maintain an accuracy better than $1 \%$ for all conditions. The flow rate of air entering the co-flow nozzle is monitored by a thermal mass flow meter (FCI STP100) and is maintained within $\pm 10 \%$ of the desired set point.

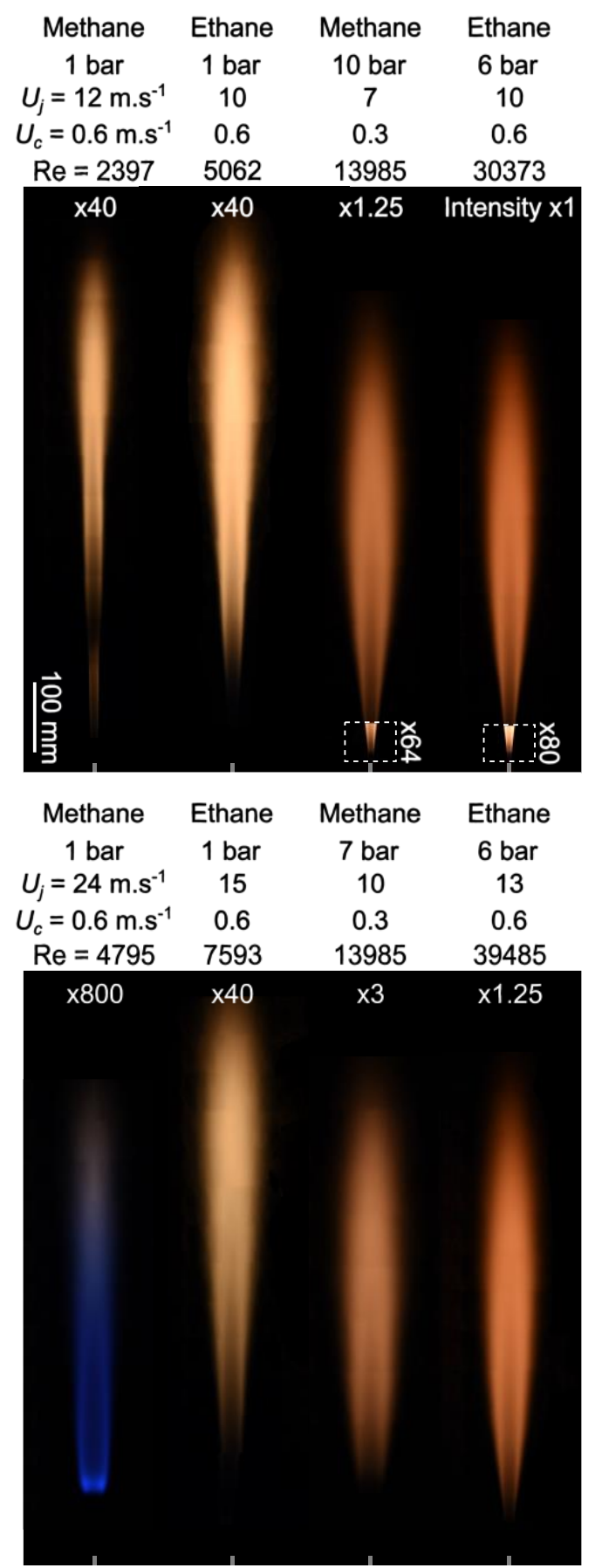

Fig. 3: Long exposure direct flame images. Images taken at different heights are stitched together. An intensity factor is provided for each image to allow comparison of the flames' luminosity. The nozzle with $t=0.58 \mathrm{~mm}$ was used for these images. 
Figure 3 shows long exposure (15 s) direct flame images recorded with a DSLR camera (Nikon D810 with AF-S Nikkor 24-120 mm lens, F/22, iso250) for eight selected operated conditions. Flames in the top row are attached and the jet velocity corresponds to $80 \%$ of the detachment jet velocity in each case. Flames in the bottom row are lifted. Because flames are much longer than the 150-mm diameter side windows, the burner is mounted on a vertical translation stage and a collection of images is recorded at different successive burner heights before being stitched together to yield the images shown in Fig. 3. Neutral density filters of various optical densities are installed in front of the camera lens in order to compensate for large differences in the flames luminosity and this is quantified by an intensity factor in Fig. 3. Figure 3 shows that pressure, fuel, and jet velocity have a large influence on the flame height and soot loading. However, these features are not the main focus of this study and comprehensive studies on effects of pressure on the flame height and soot loading of turbulent nonpremixed jet flames will be reported elsewhere. Instead, Fig. 3 is shown here only for illustration purposes.

Starting with an attached laminar flame with $U_{j}=0.5 \mathrm{~m} . \mathrm{s}^{-1}$, flame detachment is reached by progressively increasing the jet velocity $U_{j}$ in small increments of $0.20 \mathrm{~m} . \mathrm{s}^{-1}$ and is detected by eye through one of the HPCD's side windows. In each case, a minimum of five replicates are done to ensure statistical convergence of the measured detachment jet velocity $U_{d}$ and assess its variability, which was $1.6 \mathrm{~m} \cdot \mathrm{s}^{-1}$ (equivalent to $\pm 8 \%$ ) at most. Because the jet velocity is increased slowly in these experiments, thermal steady-state is always reached at the time of detachment and effects of different burner nozzle temperatures on detachment are not considered here.

\section{Results and discussion}

The physical mechanisms leading to flame detachment are examined here by means of a comprehensive parametric study. Section. 3.1 successively shows the influence of pressure ( $1 \leq P \leq 10$ bar), nozzle wall thickness $(t=0.20 \mathrm{~mm}, 0.58 \mathrm{~mm}$, and $0.89 \mathrm{~mm})$, co-flow velocity $\left(U_{c}=0.3 \mathrm{~m} \cdot \mathrm{s}^{-1}, 0.6 \mathrm{~m} \cdot \mathrm{s}^{-1}\right.$, and $\left.0.9 \mathrm{~m} \cdot \mathrm{s}^{-1}\right)$, and fuel (methane and ethane) on the measured detachment velocity. Using these data, a criterion to predict whether type I or type II detachment mechanism occurs is proposed in Sec. 3.2. In Sec. 3.3, a model to predict the detachment velocity in type II detachment is suggested. Results shown in Sec. 3.4 are used to explain how resorting to elevated pressure allows pushing attached non-premixed jet flames to higher levels of turbulence without facing lift-off or blow-off. Finally, potential effects of the burner lip temperature are briefly discussed in Sec. 3.5. 


\subsection{A parametric study of the detachment velocity}

Figure 4 shows the measured detachment velocity $U_{d}$ as a function of pressure for methane with $U_{c}=0.6 \mathrm{~m} . \mathrm{s}^{-1}$ and the three different nozzle wall thicknesses $t=0.20 \mathrm{~mm}$ (red squares), $0.58 \mathrm{~mm}$ (blue triangles), and $0.89 \mathrm{~mm}$ (black circles). It is evident that both pressure and nozzle wall thickness have a large influence on the flame detachment behavior. More specifically, the following points are noted:

- For the thinner nozzle with $t=0.20 \mathrm{~mm}$, the detachment velocity decreases monotonically with pressure. It is $U_{d}=11.6 \mathrm{~m} . \mathrm{s}^{-1}$ at $P=1 \mathrm{bar}$ and $U_{d}=3.4 \mathrm{~m} . \mathrm{s}^{-1}$ at $P=10 \mathrm{bar}$. The detachment velocity scales with $P^{-0.5}$ (see solid red trend line).

- For the thicker nozzles with $t=0.58 \mathrm{~mm}$ and $0.89 \mathrm{~mm}$, there is a non-monotonic behavior of the detachment velocity with pressure. The detachment velocity first decreases with pressure up to $P \approx 3$ bar. Then, it increases slowly with pressure up to $P=4.5$ bar or $P=3.5$ bar for $t=0.58 \mathrm{~mm}$ and $0.89 \mathrm{~mm}$, respectively. The detachment velocity finally decreases with pressure again up to $P=10 \mathrm{bar}$, albeit at a slower rate.

- Regardless of the nozzle wall thickness, there exists a critical pressure below which the detachment velocity scales with $P^{-0.5}$ (see solid trend lines). This critical pressure is $P \approx 3$ bar for $t=0.58 \mathrm{~mm}$ and $P \approx 2$ bar for $t=0.89 \mathrm{~mm}$. It is larger than $P=10$ bar for $t=0.20 \mathrm{~mm}$.

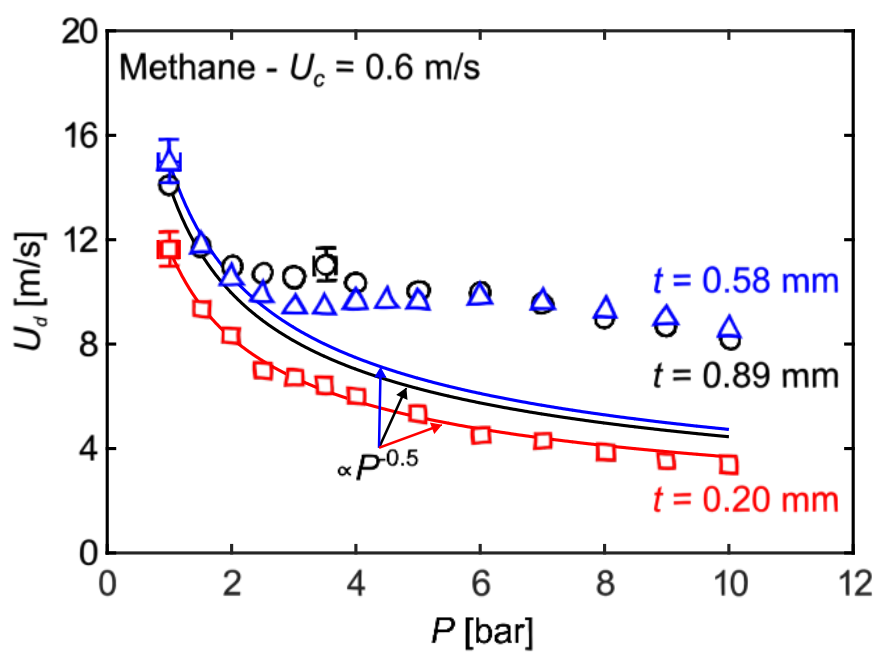

Fig. 4: Measured detachment velocity (symbols) as a function of pressure for methane and $U_{c}=0.6 \mathrm{~m} \cdot \mathrm{s}^{-1}$ and the three different nozzles. Solid lines show -0.5 power law dependencies on pressure.

It should be noted that the laminar burning velocity $S_{L}$ also scales with $P^{-0.5}$ for methane $[18,19,23$, 24]. This is due to the fact that $S_{L}$ is expected to scale with $P^{(\mathrm{n}-2) / 2}$ [18], where $\mathrm{n}$ is the overall reaction order, and $\mathrm{n}$ is close to unity for methane $[18,19,24]$. The detachment velocity is then proportional to the laminar burning velocity regardless of pressure if the nozzle is very thin $(t=0.20 \mathrm{~mm})$ or is 
proportional to the laminar burning velocity regardless of the nozzle wall thickness if the pressure is low enough ( $P \leq 2$ bar). This corroborates the previous findings of [6] that the aerodynamic type I detachment mechanism is associated with the "thin" nozzle regime.

The non-monotonic behavior of the detachment velocity with pressure for the two thicker nozzles suggests that flames exhibit a change in their detachment mechanism as pressure is increased. This is consistent with the fact that the representative flame thickness, whether it is the laminar flame thickness or the quenching distance, decreases with pressure [18-20, 25-27] and that a nozzle with a fixed wall thickness may be considered "thin" in comparison to this representative flame thickness at $P=1$ bar but becomes "thick" above a higher critical pressure.

Figure 5 shows the measured detachment velocity $U_{d}$ as a function of pressure for methane with $t=0.89 \mathrm{~mm}$ and the three different co-flow velocities $U_{c}=0.3 \mathrm{~m} . \mathrm{s}^{-1}$ (red squares), $0.6 \mathrm{~m} . \mathrm{s}^{-1}$ (black circles), and $0.9 \mathrm{~m} \cdot \mathrm{s}^{-1}$ (blue triangles). The following points are noted:

- Regardless of the co-flow velocity, there exists a pressure below which the detachment velocity scales with $P^{-0.5}$ (see solid trend lines), which is consistent with Fig. 4.

- If $P<3$ bar, the detachment velocity decreases as the co-flow velocity increases. This is consistent with the aerodynamic detachment type I observed for "thin" nozzles in [6].

- If $P \geq 3$ bar, the detachment velocity is somewhat insensitive to the co-flow velocity with discrepancies lying within error bars. Comparing these results to those of [6] suggests that flames for $P \geq 3$ bar exhibit type II detachment behavior, associated with the "thick" nozzle regime. 
In Fig. 5, effects of the co-flow depend on the pressure, which provides further evidence that pressure influences the detachment mechanism. Regardless of the nozzle wall thickness, the attached flame is never strictly anchored to the nozzle tip but sits away from it, at a distance equal to some quenching distance. Type I detachment occurs when the stream velocity at the flame base exceeds the local maximum burning velocity. The stream velocity at the flame base is a weighted function of the jet and co-flow velocities and their respective contributions are a function of the stoichiometric mixture fraction [28]. Therefore, type I detachment can be reached either by increasing the jet velocity or the co-flow velocity, which explains the findings of Fig. 5 and corroborates those of [6].

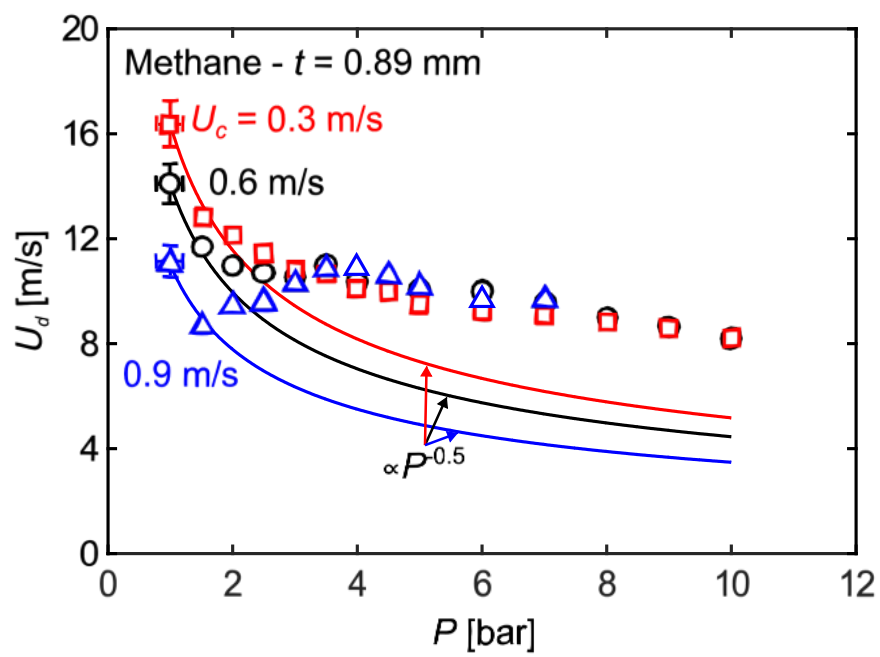

Fig. 5: Measured detachment velocity (symbols) as a function of pressure for methane and the nozzle with $t=0.89 \mathrm{~mm}$ and three different co-flow velocities. Solid lines show -0.5 power law dependencies on pressure.

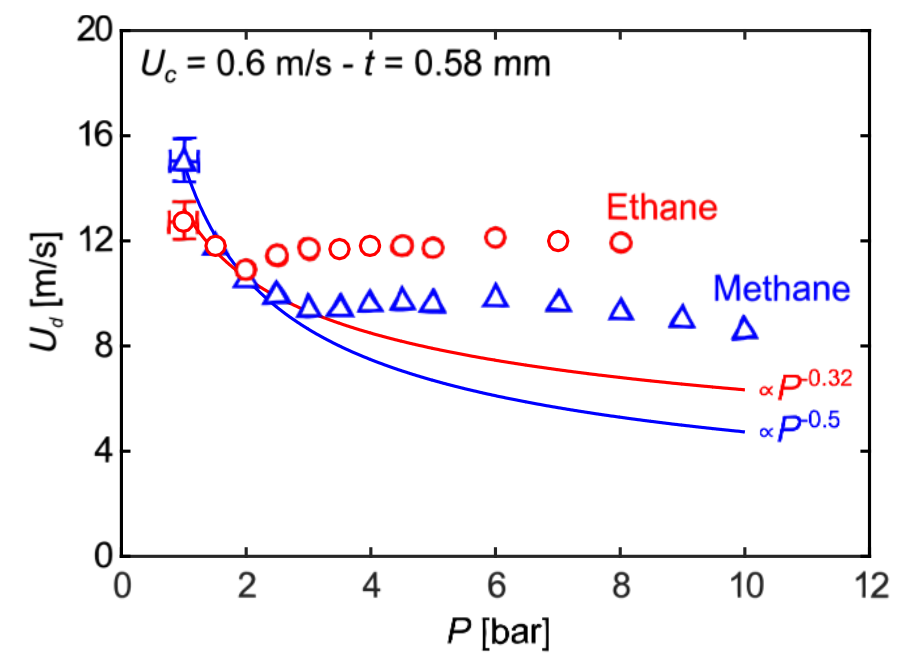

Fig. 6: Measured detachment velocity (symbols) as a function of pressure for $U_{c}=0.6 \mathrm{~m} . \mathrm{s}^{-1}$ and the nozzle with $t=0.58 \mathrm{~mm}$ and methane and ethane fuels. Solid lines show -0.5 and -0.32 power law dependencies on pressure.

In the "thick" nozzle regime, the distance between the flame base and the nozzle tip is also equal to some quenching distance but is now smaller compared to the nozzle wall thickness. The flame is then 
tucked away in the wake of the nozzle and the flame base velocity is close to zero and is much less sensitive to the jet and co-flow velocities. This is also compatible with findings of [6].

Figure 6 shows the measured detachment velocity $U_{d}$ as a function of pressure for $U_{c}=0.6 \mathrm{~m} . \mathrm{s}^{-1}$ with $t=0.89 \mathrm{~mm}$ and the two different fuels, methane (blue triangles) and ethane (red circles). Ethane exhibits a similar behavior to that of methane. The detachment velocity has a non-monotonic behavior with pressure. The detachment velocity is somewhat insensitive to pressure if $2.5 \leq P \leq 8$ bar and it decreases monotonically with pressure if $P \leq 2$ bar. Note that if $P \leq 2$ bar, the detachment velocity for ethane scales well with $P^{-0.32}$. The detachment velocity is then also proportional to the laminar burning velocity because the overall reaction order of ethane is $\mathrm{n} \approx 1.36$, yielding $S_{L} \propto P^{(\mathrm{n}-2) / 2}=P^{-0.32}$. Also note that the detachment velocity is larger for ethane than for methane if $P>2$ bar.

\subsection{A criterion to distinguish between detachment mechanisms}

For the two thicker nozzles with $t=0.58 \mathrm{~mm}$ and $0.89 \mathrm{~mm}$ and for both fuels, there exists a critical pressure $P_{\text {crit }}$ above which the detachment velocity ceases to be proportional to the laminar burning velocity. If this critical pressure is defined arbitrarily as the pressure yielding a $10 \%$ deviation from the $P^{(\mathrm{n}-2) / 2}$ trend, by data interpolation, one finds $P_{\text {crit }}=3.09$ bar for methane and $t=0.58 \mathrm{~mm}$, $P_{\text {crit }}=2.03$ bar for methane and $t=0.89 \mathrm{~mm}$, and $P_{\text {crit }}=2.13$ bar for ethane and $t=0.58 \mathrm{~mm}$. Based on the results of Sec. 3.1 and the findings of [6], it can be argued that these critical pressures should, in each case, correspond to the pressure above which the detachment mechanism switches from type I to type II and, therefore, switches from the "thin" to the "thick" nozzle regime. This can be verified by comparing the representative flame thickness to the nozzle wall thickness at each respective critical pressure. Note that the determining quantity is, in fact, the flame's stand-off distance $[9,16]$ or quenching distance as used by [6]. However, and for convenience, a representative flame thickness is used here as a surrogate because it can be computed analytically or with accessible numerical tools.

Here, the representative flame thickness is taken equal to the laminar flame thickness $\delta_{L}$ and is computed from the temperature profile as follows: $\delta_{L}=\left(T_{b}-T_{u}\right) /\left(\partial T / \partial x_{\max }\right)$, where $T_{b}$ and $T_{u}$ are the burnt and unburnt temperatures, respectively. This is consistent with [9]. The temperature profile is obtained from the premixed laminar flame-speed calculation module available in ChemkinPro [29] and the USCII skeletal chemistry mechanism [30], which was shown to provide good performances for methane and ethane at atmospheric and elevated pressures [31]. Therefore, the laminar flame thickness is that of an unstrained laminar flame and is computed at stoichiometry to represent non-premixed combustion. Results of these simulations are showed in Fig. 7. As anticipated, the laminar flame thickness decreases with pressure for both fuels. To illustrate, the laminar flame 


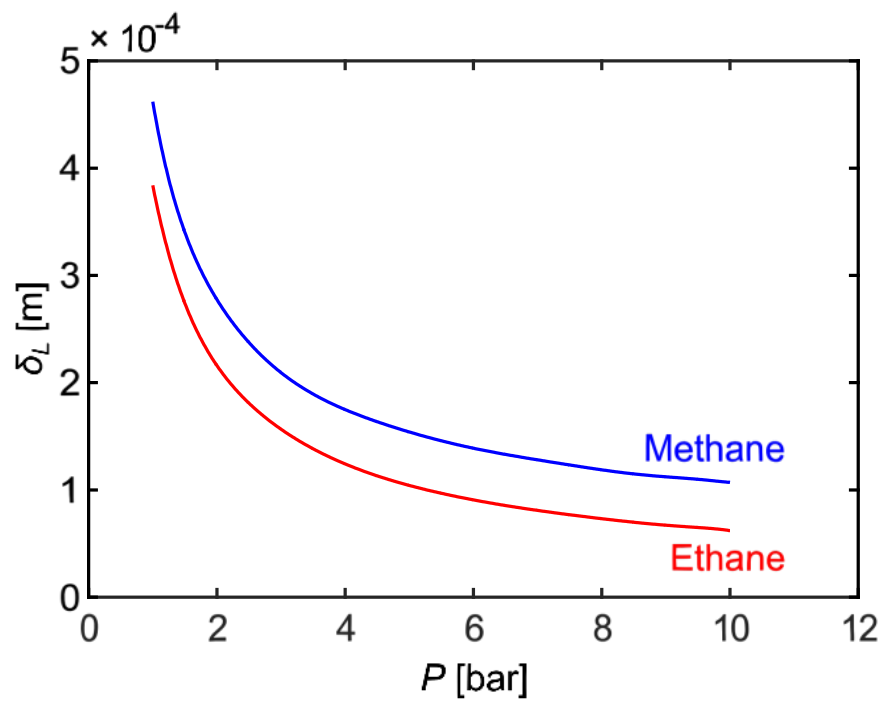

Fig. 7: Computed laminar flame thickness of an unstrained stoichiometric flame as a function of pressure for methane and ethane fuels.

thickness decreases from $\delta_{L}=0.46 \mathrm{~mm}$ at $P=1$ bar to $\delta_{L}=0.11 \mathrm{~mm}$ at $P=10$ bar for methane and decreases from $\delta_{L}=0.38 \mathrm{~mm}$ at $P=1$ bar to $\delta_{L}=0.06 \mathrm{~mm}$ at $P=10$ bar for ethane. The laminar flame thickness is globally larger for methane than for ethane.

The laminar flame thickness takes the following values at the measured critical pressures: $\delta_{L}=0.21 \mathrm{~mm}$ for methane and $t=0.58 \mathrm{~mm}, \delta L=0.28 \mathrm{~mm}$ for methane and $t=0.89 \mathrm{~mm}$, and $\delta_{L}=0.20 \mathrm{~mm}$ for ethane and $t=0.58 \mathrm{~mm}$. Therefore, the ratio of the nozzle wall thickness to the laminar flame thickness at the critical pressure, $R_{\text {crit }}=t / \delta$, is: $R_{\text {crit }}=2.8$ for methane and $t=0.58 \mathrm{~mm}$, $R_{\text {crit }}=3.2$ for methane and $t=0.89 \mathrm{~mm}$, and $R_{\text {crit }}=2.9$ for ethane and $t=0.58 \mathrm{~mm}$. A value of $R_{\text {crit }}=3.0 \pm 7 \%$ can then be used to predict the transition between type I and type II detachment mechanisms and it has been validated for two fuels and two nozzle wall thicknesses. Under the assumption that the size of the recirculation zone in the wake of a nozzle of wall thickness $t$ is comparable to $t$ [32], the value $R_{c r i t}=3.0$ makes physical sense. Indeed, for a flame to be tucked-away and protected within the recirculation zone, the distance between the flame base and the nozzle, for which the laminar flame thickness provides a reasonable approximation, should be significantly smaller than the size of the recirculation zone. One can argue that a distance equal to $1 / 3$ of the recirculation zone size satisfies this criterion.

For methane and the tapered nozzle with $t=0.20 \mathrm{~mm}, t / \delta_{L}=1.87<3.0$ at $P=10$ bar. This confirms why transition from type I to type II detachment was not observed for the tapered nozzle with $t=0.20 \mathrm{~mm}$ over the examined pressure range. Such transition is expected to occur around $P=23$ bar. 
Data show that a critical ratio $t / \delta L=3.0$ separates type I and type II detachment mechanisms if $\delta_{L}$ is defined using unstrained premixed flame characteristics. Another definition of $\delta L$, based on a quenching or a stand-off distance, would have led to a slightly different critical ratio but would have not altered the conclusions of this study.

\subsection{A model to predict detachment by local flame extinction}

The $R_{c r i t}=3.0$ criterion marks the nominal transition threshold from type I to type II detachment. For type I, the detachment velocity is proportional to the laminar burning velocity meaning that the detachment velocity can be predicted for any specified pressure as long as it is known for at least one pressure and $t / \delta_{L}$ remains significantly below $R_{c r i t}=3.0$. The objective of this subsection is to propose a model to also predict the detachment velocity for type II detachment if $t / \delta_{L} \geq 3.0$.

Type II detachment is triggered by local extinction of the flame front at a breakpoint usually located slightly downstream of the nozzle [6]. This means that, locally and at the time of detachment, the strain rate experienced by the flame front exceeds the extinction strain rate, which is a function of fuel and pressure [31]. In other words, the time scale of the flow feature responsible for flame extinction becomes smaller that the characteristic chemical time scale of the flame at the location of extinction [11]. In this study, the characteristic chemical time scale is taken equal to the inverse of the extinction strain rate $\kappa_{\text {ext }}$ [4]. The extinction strain rate is computed using the extinction of diffusion opposedflow flame module available in Chemkin-Pro [29] and the USCII skeletal chemical mechanism [30]. The USCII mechanism has been shown to capture reasonably the extinction strain rate for nitrogendiluted methane and ethane fuels at pressures up to $P=10$ bar [31]. Another valid approach would have been to compute the flame's chemical time scale using a perfectly stirred reactor because this has been shown to yield very similar numbers over wide ranges of equivalence ratios and pressures [4]. Results are shown in Fig. 8. For both fuels, the extinction strain rate increases with pressure,

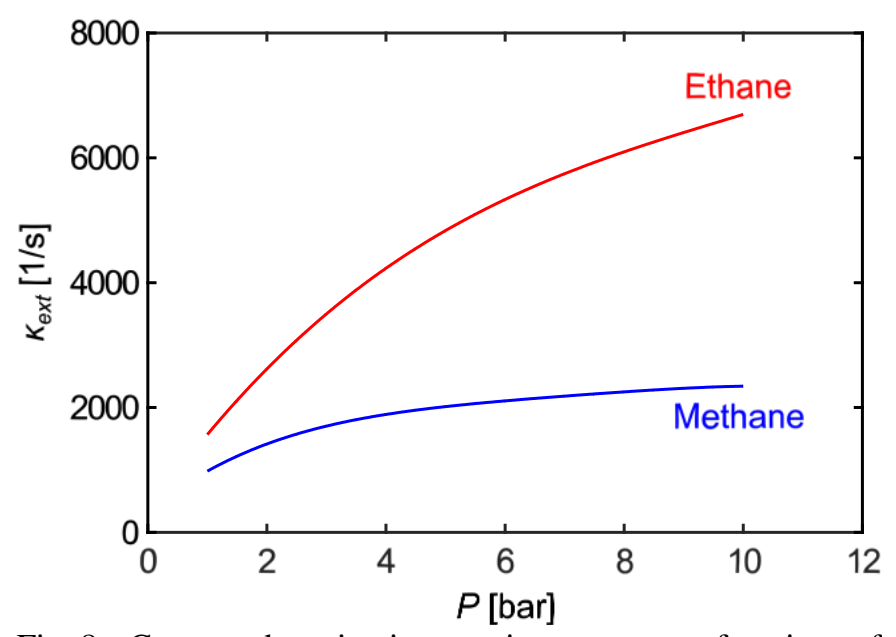

Fig. 8: Computed extinction strain rate as a function of pressure for methane and ethane fuels. 
which is consistent with previous observations [31, 33]. To illustrate, the extinction strain rate increases from $\kappa_{e x t}=980 \mathrm{~s}^{-1}$ at $P=1$ bar to $\kappa_{e x t}=2341 \mathrm{~s}^{-1}$ at $P=10$ bar for methane. The extinction strain rate is globally larger for ethane. It increases from $\kappa_{e x t}=1567 \mathrm{~s}^{-1}$ at $P=1$ bar to $\kappa_{e x t}=6695 \mathrm{~s}^{-1}$ at $P=10$ bar.

At this point, it is not clear which flow time scale controls local flame extinction and detachment in these turbulent non-premixed jet flames. One can define four main flow time scales:

- The bulk flow time scale, $\tau_{D}$, which relates to the nozzle inner diameter $D$ and the bulk jet velocity $U_{j}$ as follows: $\tau_{D}=D / U_{j}$.

- The integral time scale, $\tau$, which relates to the integral scale of turbulence $l$ and the turbulent fluctuations of the velocity $u^{\prime}$ as follows: $\tau_{l}=l / u^{\prime}$ [34]. Based on [35], It is reasonable to consider that $\tau_{D}$ and $\tau_{l}$ are comparable and at least proportional.

- The Taylor time scale, $\tau_{\lambda}$, which relates to the Taylor scale of turbulence $\lambda$. The Taylor time scale may be evaluated from the bulk flow time scale using the following expression: $\tau_{\lambda} \propto$ $\tau_{D} \operatorname{Re}_{D}{ }^{-0.5}[36,37]$.

- The Kolmogorov time scale, $\tau_{\eta}$, which relates to the Kolmogorov scale of turbulence $\eta$. The Kolmogorov time scale may also be evaluated from the bulk flow time scale using the expression $\tau_{\eta} \propto \tau_{D} \operatorname{Re}_{D}{ }^{-0.5}[36,37]$ and is therefore proportional to the Taylor time scale.

Figure 9a shows the chemical time scale plotted as a function of the bulk flow time scale at detachment for all the conditions examined in this study with $t / \delta_{L} \geq 3.0$. It is evident that there is no collapse of the data, which confirms that the bulk flow scale is not the scale controlling local extinction and detachment. This is reasonable since this scale does not account for effects of turbulence. Because the integral time scale is proportional to the bulk flow time scale, Fig. 9a also shows that the integral scale of turbulence does not control local extinction and detachment. 

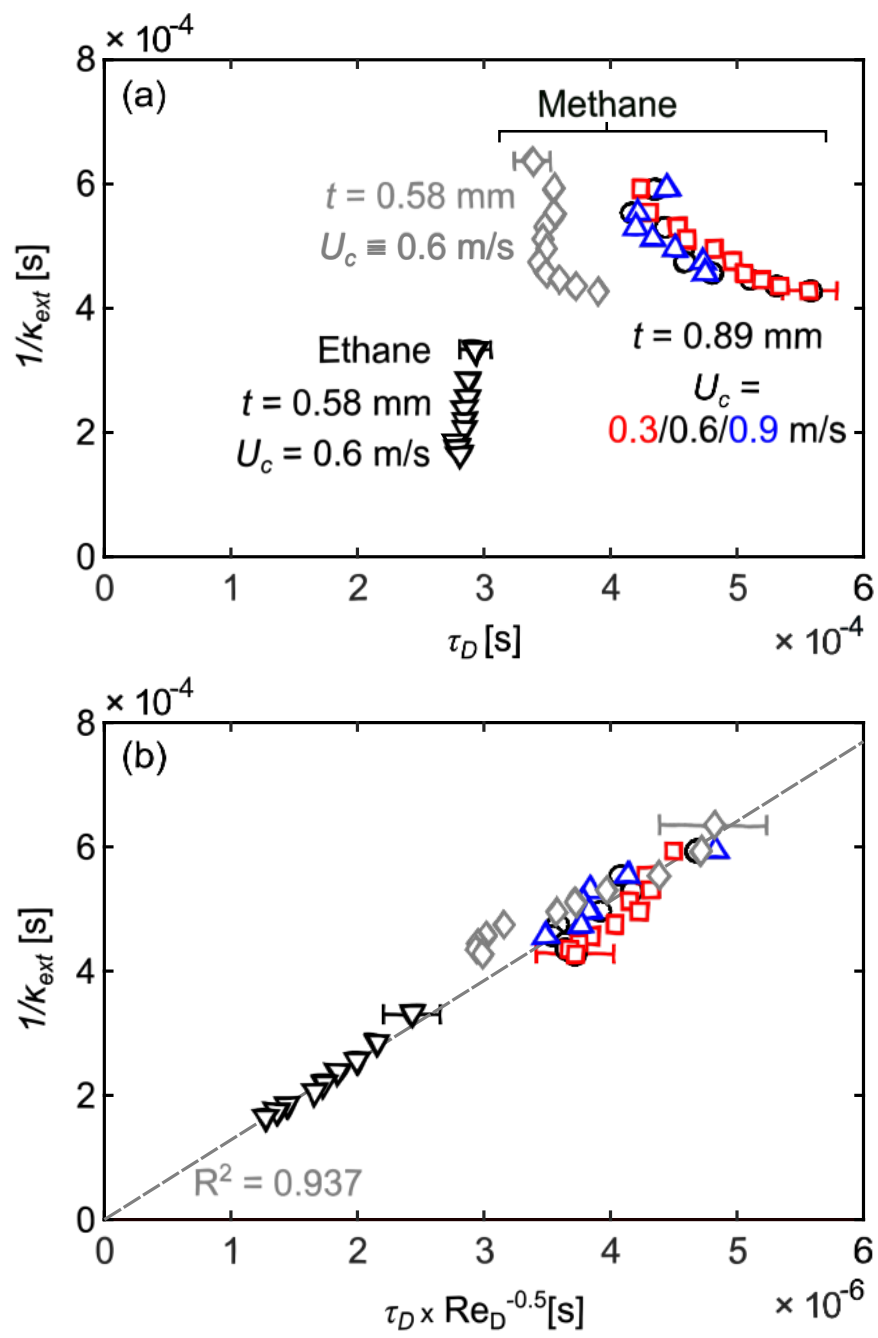

Fig. 9: (a) Inverse of the computed extinction strain rate as a function of the bulk flow time scale $\tau_{D}$ at detachment for all the data points in the "thick" nozzle regime $\left(t / \delta_{L} \geq 3.0\right)$. (b) same but as a function of $\tau_{D \times} \mathrm{Re}_{\mathrm{D}}{ }^{-0.5}$ at detachment.

Figure $9 \mathrm{~b}$ shows the chemical time scale plotted as a function of $\tau_{D} \operatorname{Re}_{D}{ }^{-0.5}$ at detachment, which is proportional to both the Taylor and Kolmogorov time scales, for all the conditions examined in this study with $t / \delta_{L} \geq 3$.0. This expression now provides a good linear fit of the data with a coefficient of determination $\mathrm{R}^{2}=0.937$. This means that both the Taylor and Kolmogorov time scales are proportional to the flame chemical time scale at extinction. Because the exact Taylor and Kolmogorov time scales are unknown and because Fig. $9 \mathrm{~b}$ warrants proportionality but not equality between chemical and flow time scales, more information is needed to determine which of the Taylor or Kolmogorov scale controls extinction.

In a turbulent flow, it is possible to estimate the time scale of any turbulent scale from the properties of the integral scale following Kolmogorov's turbulence theory. The Kolmogorov time scale may be expressed as follows: $\tau_{\eta}=\tau_{l}(\eta / l)^{2 / 3}$. Since Kolmogorov eddies are the smallest eddies found in a turbulent flow, this expression shows that the time scale of the Kolmogorov eddies is the smallest as 
well. As a consequence, in the turbulent non-premixed jet flames studied here, the Kolmogorov time scale is the first one reaching values comparable to the chemical time scale when the bulk jet velocity is progressively increased, implying that the Kolmogorov scale must be responsible for local extinction. This explains why the detachment data shown in Fig. 9b provide a good collapse when the Kolmogorov time scale is used to express the flow time scale. This result corroborates that of Yoshida et al. [14] who found that accounting for the contribution of turbulence in the total strain rate using the Kolmogorov time scale provides a good collapse of their extinction data for methane and propane fuels in a diffusion counter-flow configuration. It is then possible to conclude that the Kolmogorov scale of turbulence is the regulating scale for local flame extinction and type II detachment in turbulent non-premixed jet flames. This has been validated for a range of pressures, fuels, and co-flow velocities. The detachment velocity should then be predictable for all other pressures and fuels and any nozzle wall thickness as long as the extinction strain rate can be determined accurately and $t / \delta_{L} \geq 3.0$.

Such statement is not expected to hold for configurations involving turbulent premixed flames. Indeed, experiments conducted in turbulent premixed Bunsen flames [15] have shown that, instead, the Taylor scale of turbulence is the regulating scale for flame front and turbulence interactions. This is because Kolmogorov eddies are usually too small to induce local extinction in premixed flames, which are less sensitive to strain than non-premixed flames and are not being thinned by straining turbulent eddies.

When studying the structure and extinction of turbulent non-premixed flames, it is useful to resort to the concept of scalar dissipation rate [38]. However, determining the local scalar dissipation rate requires knowledge of the mixture fraction field, which is very challenging to measure in nonpremixed flames at elevated pressure and is, therefore, not available here. However, based on Fig. 9b, the strain rate experienced by each flame at the time and location of local extinction is known. Because values of the extinction strain rate have been obtained numerically using a non-premixed opposed flow configuration, it is possible to compute the corresponding critical scalar dissipation rate $\chi_{q}$ using the following expression: $\chi_{q}=2 a_{\text {ext }} / \pi \exp \left(-2\left[\operatorname{erfc}^{-1}\left(2 Z_{s t}\right)\right]^{2}\right)$ [38]. Here, a axt is the bulk velocity gradient at extinction and $Z_{s t}$ is the stoichiometric mixture fraction $\left(Z_{s t}=0.055\right.$ and 0.059 for methane and ethane, respectively). Predicted values of $\chi_{q}$ are deemed equivalent to the scalar dissipation rate locally experienced by the non-premixed jet flames at detachment. Table 1 summarizes the extinction strain rate and critical scalar dissipation rate for some of the conditions examined here. 
Tab. 1: Computed extinction strain rate and critical scalar dissipation rate for methane and ethane at different pressures.

\begin{tabular}{ccccccccc}
\hline$\kappa_{\text {ext }}\left[\mathrm{s}^{-1}\right]$ & $\chi_{q}\left[\mathrm{~s}^{-1}\right]$ & \multicolumn{2}{c}{1 bar } & \multicolumn{2}{c}{3 bar } & \multicolumn{2}{c}{5 bar } & \multicolumn{2}{c}{ bar } \\
\hline Methane & 976 & 14.5 & 1,702 & 25.8 & 2,015 & 30.6 & 2,188 & 33.3 \\
Ethane & 1,577 & 26.5 & 3,506 & 59.7 & 4,841 & 82.7 & 5,715 & 97.8 \\
\hline
\end{tabular}

The value of the critical scalar dissipation rate obtained for methane at $P=1$ bar agrees well with that obtained in $[39,40]$. The trend of critical scalar dissipation rate as a function of fuel is consistent with that observed in [41]. Regardless of the fuel, the critical scalar dissipation rate increases with pressure, which is also consistent with findings of [42].

\subsection{Pressure as a tool to study high Reynolds number flames}

It is also interesting to analyze the detachment data as a function of pressure in light of the bulk Reynolds number at detachment $\operatorname{Re}_{\mathrm{d}}$ instead of the bulk jet velocity at detachment $U_{d}$. This is done in Fig. 10 for all the fuel, pressure, nozzle wall thickness, and co-flow velocity conditions investigated. Globally, the bulk Reynolds number at detachment increases with pressure. This increase is close to linear for ethane and is sub-linear for methane. The rate of increase is the smallest for methane and the thin nozzle $(t=0.20 \mathrm{~mm})$, corresponding to type I detachment. For methane, the rate of increase is largest for $t=0.89 \mathrm{~mm}$ and the bulk Reynolds number at detachment increases from $\operatorname{Re}_{\mathrm{d}}=4,400$ at $P=1$ bar to $\operatorname{Re}_{\mathrm{d}}=22,400$ at $P=10$ bar. For ethane and $t=0.58 \mathrm{~mm}$, the bulk Reynolds number at detachment increases from $\operatorname{Re}_{\mathrm{d}}=6,400$ at $P=1$ bar to $\operatorname{Re}_{\mathrm{d}}=48,300$ at $P=10$ bar.

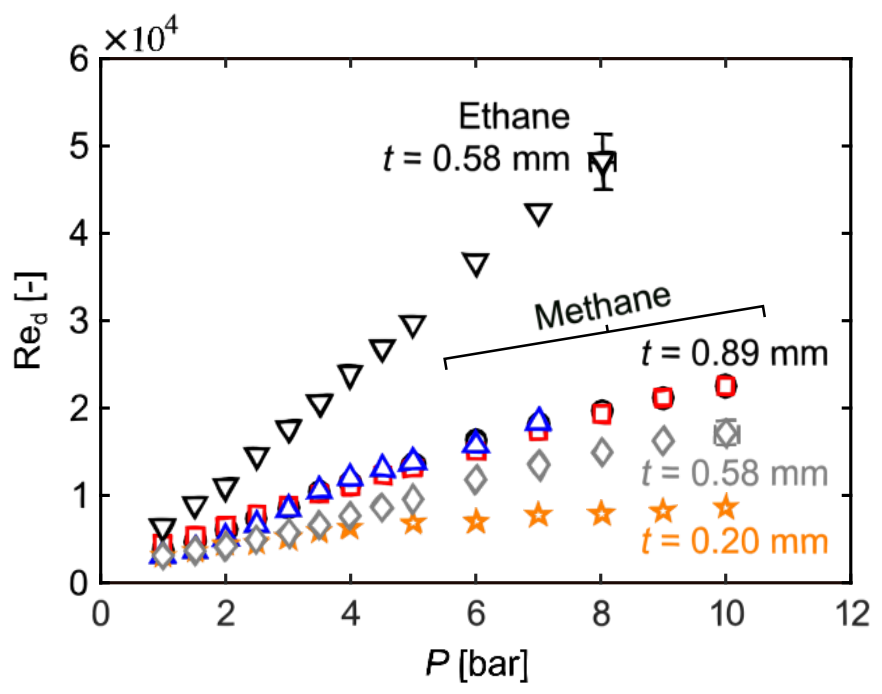

Fig. 10: Bulk Reynolds number at detachment as a function of pressure for all the conditions investigated in this study.

Using the findings of Secs. 3.1 and 3.3, the bulk jet velocity at detachment $U_{d}$ depends on the ratio of the nozzle wall thickness to the laminar flame thickness $t / \delta_{L}$ and may be expressed as follows if $t / \delta L<3.0:$ 


$$
U_{d}=f\left(t, U_{c}\right) S_{L} \quad \text { if } t / \delta_{L}<3.0
$$

where $f$ is an undefined function of $t$ and $U_{c}$ or as follows if $t / \delta_{L} \geq 3.0$ :

$$
U_{d}=128 D \operatorname{Re}_{\mathrm{d}}^{-\frac{1}{2}} \kappa_{\text {ext }} \quad \text { if } t / \delta_{L} \geq 3.0
$$

which can be rearranged as follows if $U_{d}$ is introduced in $\operatorname{Re}_{\mathrm{d}}$ :

$$
U_{d}=25.4(D v)^{\frac{1}{3}} \kappa_{\text {ext }}{ }^{\frac{2}{3}} \quad \text { if } t / \delta_{L} \geq 3.0 .
$$

where $v$ is the kinematic viscosity. Assuming that the extinction strain rate may be expressed as a function of pressure as follows: $\kappa_{\text {ext }} \propto P^{\alpha}$, the bulk Reynolds number at detachment Red then scales with pressure as follows:

$$
\begin{array}{cr}
\mathrm{Re}_{\mathrm{d}} \propto P^{\frac{n}{2}} & \text { if } t / \delta_{L}<3.0 \\
\operatorname{Re}_{\mathrm{d}} \propto P^{\frac{2(1+\alpha)}{3}} & \text { if } t / \delta_{L} \geq 3.0
\end{array}
$$

Therefore, the bulk Reynolds number at detachment increases with pressure if $t / \delta_{L}<3.0$ and $\mathrm{n}>0$ or if $t / \delta_{L} \geq 3.0$ and $\alpha>-1$. Condition $n>0$ is always met. Condition $\alpha>-1$ is met for the fuel and pressure ranges examined here (see Fig. 8) with $\alpha \approx 0.45$ and $\alpha \approx 0.22$ for methane and $1 \leq P \leq 5$ bar and $5<P \leq 10$ bar, respectively and $\alpha \approx 0.70$ and $\alpha \approx 0.46$ for ethane and $1 \leq P \leq 5$ bar and $5<P \leq 10$ bar. This explains the trends observed in Fig. 10 and implies that it is possible to stabilize attached turbulent non-premixed jet flames with much higher Reynolds numbers by resorting to elevated pressure without the need for complex flame stabilization strategies such as pilot flames, swirl, or oxygen/hydrogen enrichment. This is useful in order to study flame-turbulence interactions at conditions relevant to practical applications while conserving canonical and simple burner configurations that are amenable to diagnostics and modeling.

Based on Eq. (5), this statement is not valid for fuel and pressure conditions where $\alpha \leq-1$. To the best of the authors knowledge, such conditions are not reported in the literature. However, negative values of $\alpha$, meaning that the extinction strain rate decreases with pressure, are reported in [31] and [33]. For non-premixed flames [31], a value $\alpha=-0.31$ may be inferred from experiments with nitrogen-diluted 
methane flames between $P=5$ bar and $P=11$ bar. Values $\alpha=-0.32$ and $\alpha=-0.19$ are obtained over a similar pressure range for nitrogen-diluted ethylene and ethane flames, respectively. For lean premixed flames [33], values $\alpha=-0.17$ and $\alpha=-0.23$ are obtained numerically between $P=5$ bar and $P=10$ bar for methane and propane flames, respectively.

\subsection{Effects of the burner lip temperature}

For all the conditions considered in this study, thermal steady-state was reached before the time of detachment meaning that the tip of the burner nozzle and the incoming reactants may have been heated to some temperature above $298 \mathrm{~K}$. While accurate measurements of temperature at the burner tip have not been made, it is possible that the flame properties controlling detachment, such as the laminar burning velocity, may have changed, influencing detachment. To verify this point, additional experiments have been conducted for $P=1$ bar, $U_{c}=0 \mathrm{~m} \cdot \mathrm{s}^{-1}$, and two different burner nozzles that feature the same wall thickness $t=0.75 \mathrm{~mm}$ but are composed of two different materials, namely, stainless-steel and copper. The detachment velocities are $U_{d}=15.2 \mathrm{~m} . \mathrm{s}^{-1}$ and $U_{d}=12.9 \mathrm{~m} . \mathrm{s}^{-1}$, respectively. Switching the burner material from stainless-steel to copper has led to a decrease of $15 \%$ of the detachment velocity. Because the thermal conductivity of copper is much larger than that of stainless-steel, such decrease can be attributed to the enhanced conductive heat transfers that reduce the burner lip temperature in comparison to the stainless-steel case and, in turn, reduce the temperature at the flame base location. This confirms that, in type I detachment, heat transfers to the burner tip play a role in detachment and that some preheating of the reactants occurs. Therefore, controlling parameters, such as the laminar burning velocity, must be computed at the relevant temperature, function of the burner nozzle material and dimensions. However, due to the large temperature gradients near the flame base, the relevant temperature could not be measured accurately at this point. Regardless, using a fine gage unsheathed thermocouple (Type K, 75- $\mu \mathrm{m}$ bead diameter), it was possible to bracket the range of possible burner lip temperatures between $350 \mathrm{~K}$ and $600 \mathrm{~K}$.

This suggests that, for type I detachment, accurate prediction of the detachment velocity is difficult because it requires modeling complex heat transfers. However, as shown in Figs. 4-6, once the detachment velocity is known for one pressure, it can be predicted for other pressures by scaling with the laminar burning velocity, as long as $t / \delta_{L}<3.0$. In type II detachment "local flame extinction lifting", the breakpoint is usually located slightly downstream of the flame base and away from solid boundaries [6]. Therefore, effects of heat transfers to the burner lip are not excepted to play a large role. 
It is also possible that the burner lip temperature affects the flame's quenching distance and, in turn, influences the critical $t / \delta_{L}$ ratio that separates type I and II detachment mechanisms. For the aforementioned burner lip temperature range, it is known that the quenching distance is a weak function of temperature [43] and that it can vary by about $\pm 10 \%$. Therefore, a critical ratio $t / \delta L=3.0 \pm 0.3$ may be used to accommodate a larger range of burner nozzle materials.

\section{Conclusions}

The mechanisms underlying the detachment of turbulent non-premixed jet flames were examined experimentally at atmospheric and elevated pressures for different fuels, nozzle dimensions, and coflow velocities. The main findings are:

- Pressure and nozzle wall thickness have a large influence on the value of the detachment jet velocity.

- Regardless of fuel and co-flow velocity, if the ratio of the nozzle wall thickness to the laminar flame thickness is significantly smaller than 3.0 0.3 ("thin" nozzle regime), type I detachment occurs (flame base stability lifting). In this case, the detachment velocity is controlled by the laminar burning velocity and decreases with pressure.

- If the ratio of the nozzle wall thickness to the laminar flame thickness is equal or larger than 3 ("thick” nozzle regime), type II detachment occurs (local flame extinction lifting). In this case, the detachment velocity is not a function of the co-flow velocity and is controlled by the extinction strain rate.

- The Kolmogorov scale of turbulence is the regulating scale for local extinction and type II detachment in turbulent non-premixed jet flames. If the extinction strain rate can be determined accurately, the detachment velocity in type II detachment can be predicted for any specified fuel, pressure, and nozzle wall thickness with $U_{d}=25.4(D v)^{1 / 3} \kappa_{\text {ext }}{ }^{2 / 3}$.

- Resorting to elevated pressure allows stabilizing turbulent non-premixed jet flames with higher Reynolds numbers without facing lift-off or blow-off and without the need for more complex stabilization strategies such as pilot flames, swirl, or oxygen/hydrogen enrichment. This is useful to study flame-turbulence interactions at practically relevant conditions while conserving simple burner configurations that are suitable for diagnostics and modeling.

\section{Acknowledgements}

The research reported in this publication was supported by funding from King Abdullah University of Science and Technology (KAUST). 


\section{References}

[1] S. Su, J.H. Pohl, D. Holcombe, J.A. Hart, Techniques to determine ignition, flame stability and burnout of blended coals in p.f. power station boilers, Prog. Energy Combust. Sci. 27(1) (2001) $75-98$.

[2] K.M. Lyons, Toward an understanding of the stabilization mechanisms of lifted turbulent jet flames: Experiments, Prog. Energy Combust. Sci. 33(2) (2007) 211-231.

[3] Y. Huang, V. Yang, Dynamics and stability of lean-premixed swirl-stabilized combustion, Prog. Energy Combust. Sci. 35(4) (2009) 293-364.

[4] S.J. Shanbhogue, S. Husain, T. Lieuwen, Lean blow off of bluff body stabilized flames: Scaling and dynamics, Prog. Energy Combust. Sci. 35(1) (2009) 98-120.

[5] J. O'connor, V. Acharya, T. Lieuwen, Transverse combustion instabilities: Acoustic, fluid mechanic, and flame processes, Prog. Energy Combust. Sci. 49 (2015) 1-39.

[6] F. Takahashi, W.J. Schmoll, Lifting criteria of jet diffusion flames, Symp. (Int.) Combust. 23(1) (1991) 677-683.

[7] B. Lewis, G. Von Elbe, Chapter VII - Air entrainment and burning of jets of fuel gases, in Combustion, Flames and Explosions of Gases (Third Edition). 1987, Academic Press: San Diego. p. 479-531.

[8] F. Takahashi, M. Mizomoto, K. Tsuruyama, Stability limits of hydrogen/air coflow jet diffusion flames, 28th Aerospace Sciences Meeting (1990).

[9] M. Juniper, S. Candel, Edge diffusion flame stabilization behind a step over a liquid reactant, J. Propul. Power 19(3) (2003) 332-341.

[10] J. Hwang, N. Bouvet, K. Sohn, Y. Yoon, Stability characteristics of non-premixed turbulent jet flames of hydrogen and syngas blends with coaxial air, Int. J. Hydrogen Energy 38(12) (2013) 5139-5149.

[11] M. Akbarzadeh, M. Birouk, Near-field characteristics of a rectangular jet and its effect on the liftoff of turbulent methane flame, J. Eng. Gas Turbines Power 137(8) (2015) 081502-081502081508.

[12] M. Marin, F. Baillot, Experimental study of the lifting characteristics of the leading-edge of an attached non-premixed jet-flame: Air-side or fuel-side dilution, Combust. Flame 171 (2016) 264-280.

[13] T. Takeno, Y. Kotani, An experimental study on the stability of jet diffusion flame, Acta Astronaut. 2(11) (1975) 999-1008.

[14] A. Yoshida, T. Igarashi, Y. Kotani, Extinction of turbulent diffusion flames by Kolmogorov microscale turbulence, Combust. Flame 109(4) (1997) 669-681. 
[15] R. Fragner, F. Halter, N. Mazellier, C. Chauveau, I. Gökalp, Investigation of pressure effects on the small scale wrinkling of turbulent premixed Bunsen flames, Proc. Combust. Inst. 35(2) (2015) 1527-1535.

[16] Y. Otakeyama, T. Yokomori, M. Mizomoto, Stability of $\mathrm{CH}_{4}-\mathrm{N}_{2} /$ air jet diffusion flame for various burner rim thicknesses, Proc. Combust. Inst. 32(1) (2009) 1091-1097.

[17] S. Bang, B.J. Lee, S.H. Chung, Effect of pressure on the characteristics of lifted flames, Proc. Combust. Inst. (2018), https://doi.org/10.1016/j.proci.2018.06.094.

[18] F.N. Egolfopoulos, C.K. Law, Chain mechanisms in the overall reaction orders in laminar flame propagation, Combust. Flame 80(1) (1990) 7-16.

[19] G. Rozenchan, D.L. Zhu, C.K. Law, S.D. Tse, Outward propagation, burning velocities, and chemical effects of methane flames up to 60 ATM, Proc. Combust. Inst. 29(2) (2002) 14611470.

[20] W.R. Boyette, T.F. Guiberti, G. Magnotti, W.L. Roberts, Structure of turbulent nonpremixed syngas flames at high pressure, Proc. Combust. Inst. (2018), https://doi.org/10.1016/j.proci.2018.09.004.

[21] F. Di Sabatino, T.F. Guiberti, W.R. Boyette, W.L. Roberts, J.P. Moeck, D.A. Lacoste, Effect of pressure on the transfer functions of premixed methane and propane swirl flames, Combust. Flame 193 (2018) 272-282.

[22] T.F. Guiberti, W.R. Boyette, W.L. Roberts, A.R. Masri, Pressure effects and transition in the stabilization mechanism of turbulent lifted flames, Proc. Combust. Inst. (2018), https://doi.org/10.1016/j.proci.2018.08.033.

[23] F.N. Egolfopoulos, P. Cho, C.K. Law, Laminar flame speeds of methane-air mixtures under reduced and elevated pressures, Combust. Flame 76(3) (1989) 375-391.

[24] E. Hu, X. Li, X. Meng, Y. Chen, Y. Cheng, Y. Xie, Z. Huang, Laminar flame speeds and ignition delay times of methane-air mixtures at elevated temperatures and pressures, Fuel 158 (2015) $1-10$.

[25] M. Bellenoue, T. Kageyama, S.A. Labuda, J. Sotton, Direct measurement of laminar flame quenching distance in a closed vessel, Exp. Therm Fluid Sci. 27(3) (2003) 323-331.

[26] B. Boust, J. Sotton, S.A. Labuda, M. Bellenoue, A thermal formulation for single-wall quenching of transient laminar flames, Combust. Flame 149(3) (2007) 286-294.

[27] J. Sotton, B. Boust, S.A. Labuda, M. Bellenoue, Head-on quenching of transient laminar flame: Heat flux and quenching distance measurements, Combust. Sci. Technol. 177(7) (2005) 13051322.

[28] D. Han, M.G. Mungal, Simultaneous measurements of velocity and CH distributions. Part 1: jet flames in co-flow, Combust. Flame 132(3) (2003) 565-590. 
[29] Reaction Design: San Diego, CHEMKIN-PRO 15131, (2013).

[30] H. Wang, X. You, A.V. Joshi, S.G. Davis, A. Laskin, F.N. Egolfopoulos, C.K. Law, USC Mech Version II. High-temperature combustion reaction model of $\mathrm{H}_{2} / \mathrm{CO} / \mathrm{C} 1-\mathrm{C} 4$ compounds, (Last accessed: September 2018), Available from: http://ignis.usc.edu/USC_Mech_II.htm.

[31] U. Niemann, K. Seshadri, F.A. Williams, Methane, ethane, and ethylene laminar counterflow diffusion flames at elevated pressures: Experimental and computational investigations up to 2.0MPa, Combust. Flame 161(1) (2014) 138-146.

[32] E.E. Zukoski, F.E. Marble, Experiments concerning the mechanism of flame blow off from bluff bodies, Jet Propulsion Center, California Institute of Technology (1956) 205-210.

[33] E.S. Cho, S.H. Chung, T.K. Oh, Local Karlovitz numbers at extinction for various fuels in counterflow premixed flames, Combust. Sci. Technol. 178(9) (2006) 1559-1584.

[34] M. Lesieur, Turbulence in Fluids, Springer Netherlands, 2008.

[35] S.R. Tieszen, D.W. Stamps, T.J. O'hern, A heuristic model of turbulent mixing applied to blowout of turbulent jet diffusion flames, Combust. Flame 106(4) (1996) 442-466.

[36] H. Tennekes, J.L. Lumley, A first course in turbulence, The MIT Press, 1972.

[37] S.B. Pope, Turbulent Flows, Cambridge University Press, 2000.

[38] N. Peters, Turbulent Combustion, Cambridge University Press, 2000.

[39] A.R. Masri, R.W. Bilger, R.W. Dibble, The local structure of turbulent nonpremixed flames near extinction, Combust. Flame 81(3) (1990) 260-276.

[40] E. Mastorakos, A.M.K.P. Taylor, J.H. Whitelaw, Scalar dissipation rate at the extinction of turbulent counterflow nonpremixed flames, Combust. Flame 91(1) (1992) 55-64.

[41] R. Chen, R.L. Axelbaum, Scalar dissipation rate at extinction and the effects of oxygen-enriched combustion, Combust. Flame 142(1) (2005) 62-71.

[42] P.E. Lapenna, P.P. Ciottoli, F. Creta, Unsteady non-premixed methane/oxygen flame structures at supercritical pressures, Combust. Sci. Technol. 189(12) (2017) 2056-2082.

[43] K.T. Kim, D.H. Lee, S. Kwon, Effects of thermal and chemical surface-flame interaction on flame quenching, Combust. Flame 146(1) (2006) 19-28. 